Andrews University

Digital Commons @ Andrews University

Honors Theses

Undergraduate Research

4-3-2019

\title{
Neuronal and Behavioral Response Relationships in Virgin Females of Acheta Domesticus
}

Brandon Shin

Andrews University, shinb@andrews.edu

Follow this and additional works at: https://digitalcommons.andrews.edu/honors

Part of the Biology Commons

\section{Recommended Citation}

Shin, Brandon, "Neuronal and Behavioral Response Relationships in Virgin Females of Acheta Domesticus" (2019). Honors Theses. 202.

https://dx.doi.org/10.32597/honors/202

https://digitalcommons.andrews.edu/honors/202

This Honors Thesis is brought to you for free and open access by the Undergraduate Research at Digital Commons @ Andrews University. It has been accepted for inclusion in Honors Theses by an authorized administrator of Digital Commons @ Andrews University. For more information, please contact repository@andrews.edu. 


\title{
J. N. Andrews Honors Program \\ Andrews University
}

HONS 497

Honors Thesis

Neuronal and behavioral response relationships in virgin females of Acheta domesticus

\author{
Brandon Shin
}

03 April 2019

Advisor: Dr. Benjamin Navia

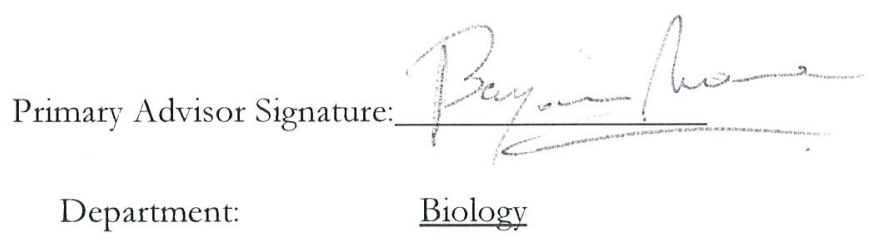




\begin{abstract}
Animals that communicate acoustically serve as models for investigating how organisms respond to sound. For instance, in field crickets, females exhibit phonotaxis (directed movement towards the sound's source) to calls with a specific frequency, intensity, and syllable period (SP) - the time between the beginning of one sound pulse and the beginning of another within the same chirp. Additionally, auditory neurons in females produce action potentials in direct response to the sound pulses of a male's call. A previous study on the species Gryllus bimaculatus demonstrated a relationship between an individual female's behavioral and neuronal responses; however, no such link between phonotactic and neuronal responsiveness has been documented in Acheta domesticus. The results of this research are consistent with the findings of the previous study; further implications are discussed.
\end{abstract}


List of Abbreviations:

CS: Calling Song

SP: Syllable Period

PTG: Prothoracic Ganglion

L3: Auditory Neuron being studied 


\section{Introduction}

Many extant animals, such as bats, whales, beetles, and butterflies, communicate via the production and reception of auditory stimuli. These stimuli can range from vocalizations to stridulations - the rubbing of specialized body parts together to produce an auditory stimulus. Within this wide population of animals, the cricket serves as a model for demonstrating how organisms respond to sound. Female crickets exhibit phonotaxis (movement in relation to the sound's source) to male calls with varying syllable periods (SPs) - the time elapsed from the onset of one sound pulse and the onset of the consecutive pulse within a single chirp set. Phonotaxis can be either positive - movement towards the source of sound, or negative movement away from, or failure to move towards, the source of sound. Male crickets produce their calling songs by rubbing their wings together. Female crickets possess tympanic membranes on their forelegs, which transmit the received song stimulus to the prothoracic ganglion - a central nerve cluster in the abdomen of the cricket which has been implicated in the processing and recognition of auditory stimuli (Huber and Thorson, 1985; Figure 3). The prothoracic ganglion relays that information to the brain via specific auditory neurons for final processing and ultimate decision making.

Experimentally identified auditory neurons in the prothoracic ganglion of crickets, such as the L3, produce action potentials in response to the sound pulses of male calling songs. These neurons are influential in the recognition of attractive conspecific male calls (Samuel et al., 2013; Stout et al., 2010; Navia et al., 2015). In addition, the particular SPs in the male's call greatly influence the responsiveness of the female; however, both behavioral and neuronal responsiveness can show large individual variability, as some crickets may be more or less 
selective than others (Stout et al., 2010). In addition, the data presented by Stout et al. (2011; in another species of cricket, Gryllus bimaculatus) indicate that although there are ranges of attractive SPs, selective responsiveness varies greatly between individuals. Female responsiveness is highly sensitive to a range of external and internal stimuli, such as the environmental temperature (Navia et al., 2015), hormone levels of the female (Choi et al., 2012), and age of the female (Stout et al., 2010). Thus, a variety of factors impact how and if an individual female responds, both behaviorally and neuronally, to a particular SP. However, Stout et al. (2011) also demonstrated that the selective responses of these females seem to show a relationship between phonotaxis and the pattern of response of the AN2 auditory neuron.

In order to demonstrate relationships in response patterns, Samuel et al. (2013) detailed how, in response to a range of SPs, the selective behaviors of an individual female (Gryllus bimaculatus) matched the selective responses of its AN2 auditory neuron. Positive phonotactic responses were characterized by the average angle of movement $\left(\leq \pm 60^{\circ}\right)$ and the net length of the path towards the sound (Samuel et al., 2013), while attractive neuronal responses were determined by the high levels of decrement - a decrease in the number of spikes produced by the neuron in response to consecutive pulses within a chirp (Stout et al., 2011).

Although Samuel et al. (2013) demonstrated a relationship between behavioral and neuronal responses to attractive calls in individual females of $G$. bimaculatus, only large patterns of behavioral and neuronal responsiveness have been documented in individual females of Acheta domesticus (Stout et al., 2010; Navia et al., 2015). This species possesses an AN2 homolog: the L3 auditory neuron (Stout et al., 2011), but there have been no studies which 
attempt to determine a relationship between the behavioral and neuronal responses in individual females of $A$. domesticus.

This study investigates individual virgin females of $A$. domesticus to document a relationship between each cricket's phonotactic behavior and L3 neuronal impulses in response to model calls. First, the phonotactic responses of individual females in response to calls with varying syllable periods will be assessed in order to determine which calls elicit positive phonotaxis in the animal. Then, the responses of the same cricket's L3 auditory neuron in response to those calls will be recorded in order to determine whether there is a relationship between phonotactic behavior and the levels of decrement in the L3 neuron. 


\section{Materials and Methods}

\section{Cricket Care}

Four week old nymphs of Acheta domesticus were obtained from Fluker's Cricket Farm (Baton Rouge, Louisiana) and were maintained in a 100-L container until molting (adulthood). Cricket chow, water, and egg cartons for shelter were provided in each container. These containers were checked daily for adults, which were subsequently removed, separated by sex (males and females), and placed into separate 16-L containers. The colonies were maintained at $22-24^{\circ} \mathrm{C}$ in a 12 hour light-dark cycle, with lights on at $0600 \mathrm{~h}$.

\section{Behavioral testing}

Virgin female crickets (6-14 days) were used for this study. First, a metal pin was attached to the back of the cricket with wax - this metal pin was then mounted onto a metal rod and the cricket was placed on a non-compensating, spherical treadmill, where phonotactic behavior was tested (Samuel et al., 2013). The treadmill was housed in a dark, soundproof, room temperature $\left(22-24^{\circ} \mathrm{C}\right)$ box in which the steel rod was immobilized, as seen in Figure 1. Crickets were left in this box for five minutes before testing, allowing them to adjust to the new environment. Computer generated model calling songs $(5 \mathrm{kHz}, 85 \mathrm{~dB})$ composed of three syllable chirps with syllable periods of $30-90 \mathrm{~ms}$ were played in a standardized nonsequential order (50, $90,70,30,60,80,40 \mathrm{~ms}$ ) through a speaker hidden in the box (Figure 1). In order to allow the insect time to rest, chirps were played for five minutes, with three minutes of silence in between different SPs. The cricket's course of movement relative to the sound's source was recorded by the computer software Optical Kugel, which tracked the treadmill rotations via two computer mice placed on the $\mathrm{X}$ and $\mathrm{Y}$ axes of the treadmill. During testing, phonotaxis was considered 
positive if the cricket's average angle of movement towards the sound's source was $\leq \pm 60^{\circ}$, and if the length of path towards the sound's source was at least twice the length of path away from the sound's source (Samuel et al., 2013).

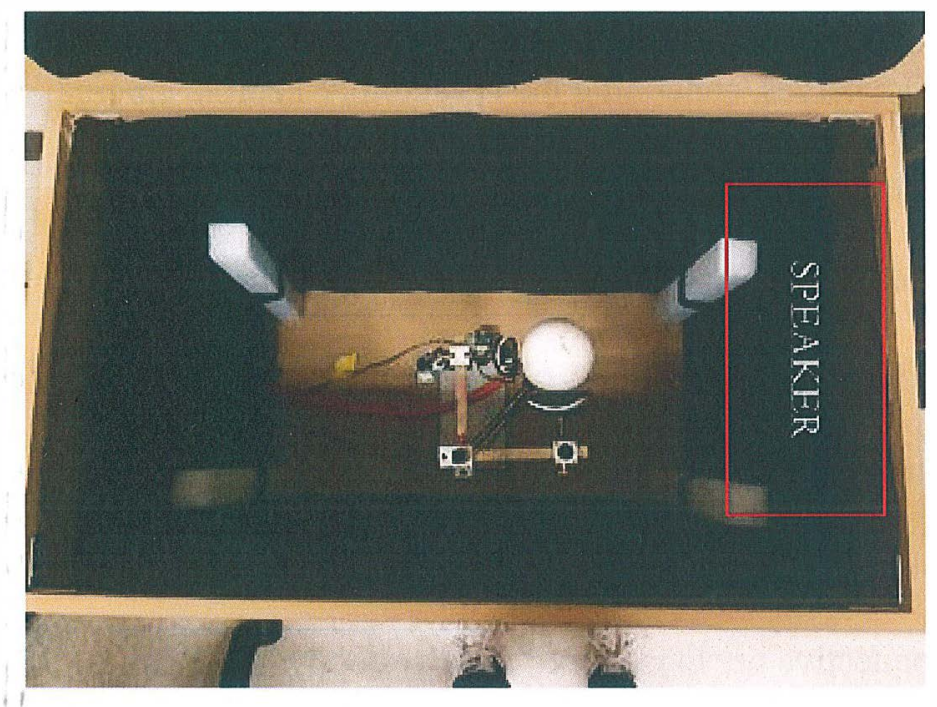

Figure 1. Superior view of the treadmill and housing used to test the cricket's phonotactic behavior. The speaker is located on the right side of the box and the metal rod is mounted onto the square peg diagonal to the treadmill.

\section{Electrophysiology}

After testing on the treadmill, each female cricket was then mounted onto a wax block, ventral side up. Wax strips were placed across its body, forming an " $\mathrm{X}$ " which immobilized the two rear pairs of legs but left the forelegs exposed. The forelegs were immobilized by placing two small U-shaped pins on top of the legs, which left the tympanic membranes in the forelegs uncovered so that the cricket could hear the model calls. Finally, a thin wax strip was used to immobilize the insect's head, preventing the cricket from moving and disrupting the experiment. In order to control for variability in responsiveness due to environmental temperature or experimental procedure, all crickets were tested at the same temperatures $\left(22-24^{\circ} \mathrm{C}\right)$ and 
underwent nearly identical behavioral testing and surgeries (Choi et al., 2012; Stout et al., 2010; Samuel et al., 2013).

A small portion of the exoskeleton on the ventral side of the cricket's thorax was removed using microscissors and forceps, revealing the prothoracic ganglion - a central nerve cluster (Figure 3). The ascending connective nerves, containing the L3 auditory neuron, emerge from the top of the prothoracic ganglion, running lengthwise towards the cricket's head.

To test the selective responses of the L3, the same set of pre-recorded calls used for behavioral testing (see above) were played through two speakers placed on both sides of the mounted cricket, one speaker per side, equally stimulating both the right and left tympanic membranes, as seen in Figure 2. A custom microsuction electrode, made from polyvinyl tubing, was then placed on top of the ascending connective nerve to record the electrophysiological responses of the L3 neuron. Saline was sprayed on top of the cricket periodically to keep the nerves moist, and a silver wire was inserted into the saline as a ground wire.

After the neuron was located, a vacuum was created via suction from the electrode, helping to isolate the L3 neuron and minimize interference from other electrical activity in the animal, allowing for more effective measurement of the L3's action potentials (B. Navia, personal communication). Each syllable period was played for 60 seconds, with 30 second breaks in between, giving the cricket time to rest. The electrical activity was recorded using a PowerLab 2/20 and analyzed via a Post-Stimulus Time (PST) histogram created in LabChart (ADInstruments; Figure 5). The histogram was used to determine the number of action potentials produced by the L3 neuron in response to the first and last sound pulses within a chirp (Figure 4). Because the males of $A$. domesticus produces chirps in sets of three, the formula 
" $\left[1-\left(n_{3} / n_{1}\right)\right] \times 100$ " was used to calculate the percentage of decrement - the decrease in the number of spikes produced by the neuron in response to the last sound pulse than to the first sound pulse within a single chirp; this value changes based on the attractiveness of the call's SP (Atkins et al., 1989; Stout et al., 2011); $\mathrm{n}_{3}$ and $\mathrm{n}_{1}$ represent the number of action potentials in the L3 in response to the third and first chirps, respectively. A higher level of decrement in response to a given SP is strongly related to the attractiveness of that particular SP (Stout et al., 2011).

\section{Characterization of the $L 3$}

The identity of the L3 neuron was verified via its encoding of 5 and $16 \mathrm{kHz}$ model calls at intensities above 70 and $60 \mathrm{~dB}$, respectively (Jeffery et al., 2005). In addition, the presence of prolonged responses to $5 \mathrm{kHz}$ calls and not to $16 \mathrm{kHz}$ calls, as well as decrement, point towards the correct characterization of the L3 (Navia et al., 2003).
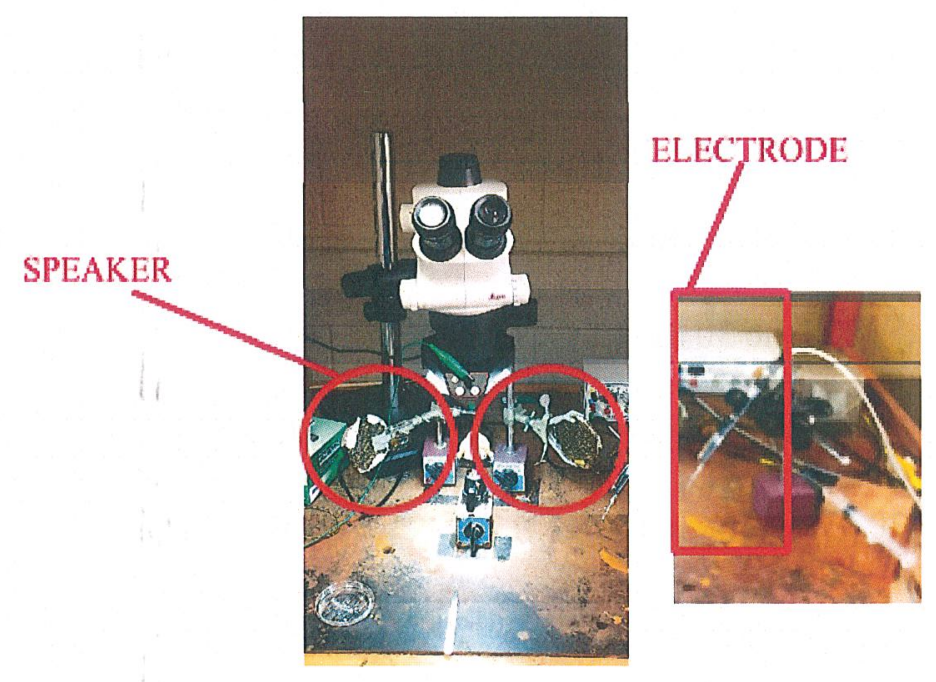

Figure 2. The surgical station containing the speakers through which the computer-generated stimuli are played, as well as the microsuction electrode through which the neuronal responses are recorded.

Statistical Analysis

The relationship of the decrement percentages between the crickets that responded with positive phonotaxis and those that responded with negative phonotaxis was determined via the 
use of an independent sample t-test. In addition, individual graphs were developed for each cricket illustrating individual relationships between phonotaxis and decrement levels (Figure 6).

\section{Results}

Seven virgin female crickets were tested in total for both phonotactic behavior and neuronal responsiveness. These females exhibited varying individual responses, both phonotactically and neuronally, to the different SPs played (Figure 6A-G). Cricket 1, a six-day old virgin female (6A), exhibited positive phonotaxis in response to 30-70 ms SPs. In addition, decrement levels remain relatively constant at approximately $30 \%$ across all syllable periods, with an increase to about $47 \%$ in response to the $70 \mathrm{~ms} \mathrm{SP}$ call, and a decrease to $21 \%$ in response to the $90 \mathrm{~ms}$ SP call. Cricket 2, a thirteen-day old virgin female (6B), failed to exhibit positive phonotaxis to any of the presented SPs. Its neuronal responses indicated a range of decrement from $4.5-23.7 \%$, providing an average decrement level of approximately $13 \%$. Cricket 3, a eight-day old virgin female (6C), also failed to exhibit positive phonotaxis in response to any of the presented SPs - it also produces very low decrement levels across all tested SPs, with a maximum level of $11.6 \%$ in response to the $30 \mathrm{~ms}$ call. Cricket 4 , a fourteen-day old virgin female (6D) also failed to exhibit positive phonotaxis in response to any SPs; however, its average decrement level of about $20 \%$ decrement (range $=7.1-25.9 \%$ ) is slightly higher than those of Cricket 2 and 3. Cricket 5, also a fourteen-day old virgin female $(6 \mathbb{E})$, exhibited positive phonotaxis in response to one SP: $40 \mathrm{~ms}$. In addition, its L3 responded with a higher level of decrement $(23 \%)$ than it did to other SPs which elicited negative phonotaxis. Cricket 6 , an eight-day old virgin female (6F), exhibited positive phonotaxis to all seven syllable periods; however, its decrement levels fluctuated in response to all SPs tested, 
ranging from $4 \%$ in response to $80 \mathrm{~ms}$, to $47 \%$ decrement in response to $50 \mathrm{~ms}$. Finally, Cricket 7, a seven-day old virgin (6G), displayed positive phonotaxis in response to four of the seven SPs tested: 30, 50, 60, and $70 \mathrm{~ms}$. Its decrement levels in response to these SPs were higher than its decrement levels in response to the SPs which elicited negative phonotaxis (17\% v. 10\%). From an overarching standpoint, the responses of the crickets ranged from a complete lack of responsiveness (positive phonotaxis to zero SPs), to total indiscriminate responsiveness (positive phonotaxis to all seven SPs). However, when all the responses of the crickets were compiled, the animals appear to respond more readily, in terms of both behavior and neuronal response, to the $30-70 \mathrm{~ms}$ SP calls (Figure 7). In addition, by utilizing an independent sample t-test, our results indicate that the L3 in crickets which respond to a particular SP with positive phonotaxis also exhibit a statistically significant higher level of decrement to those same SPs, when compared to the levels of decrement they exhibit in response to SPs which elicited negative phonotaxis (Figure $8, \mathrm{t}(47)=-4.3856, \mathrm{p}=3.24 \times 10^{-5}$ ). 


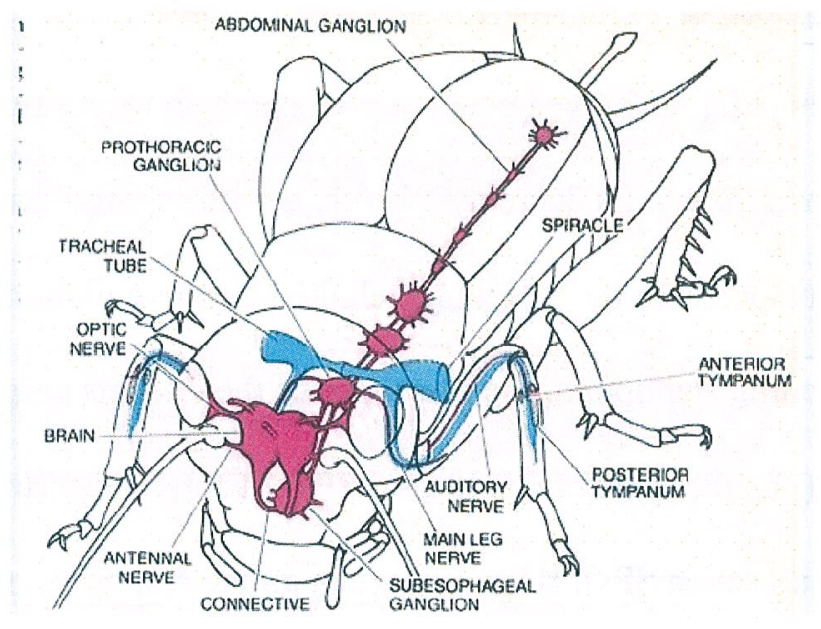

Figure 3. Diagram of the neuronal circuitry of a cricket of Acheta domesticus (Huber and Thorson, 1985). This investigation is most involved with the prothoracic ganglion and the connective nerves ascending from the ganglion towards the brain, which contain the neurons involved in processing and filtering auditory stimulus.

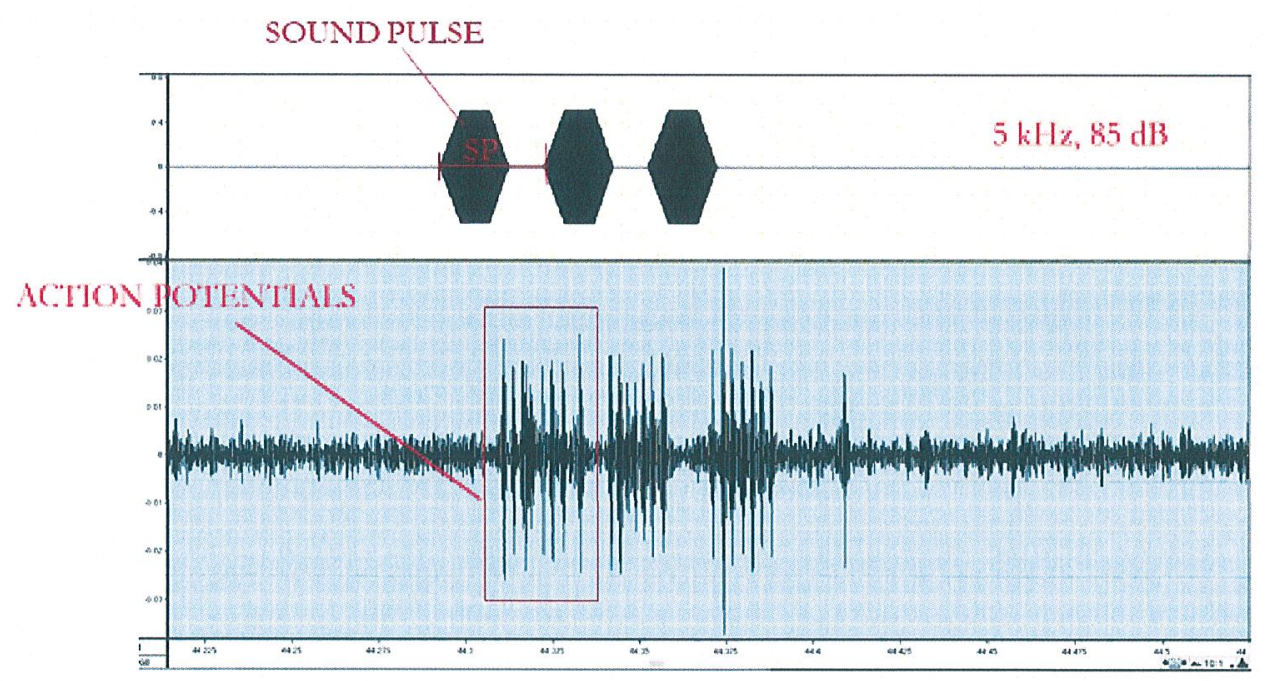

Figure 4. An example of the computer generated chirps (40 ms SP) and the cricket's neurological output (each spike represents an action potential) in response to each sound pulse. The length of time which elapses from the onset of the first pulse to the onset of the next indicates a single syllable period (SP). 


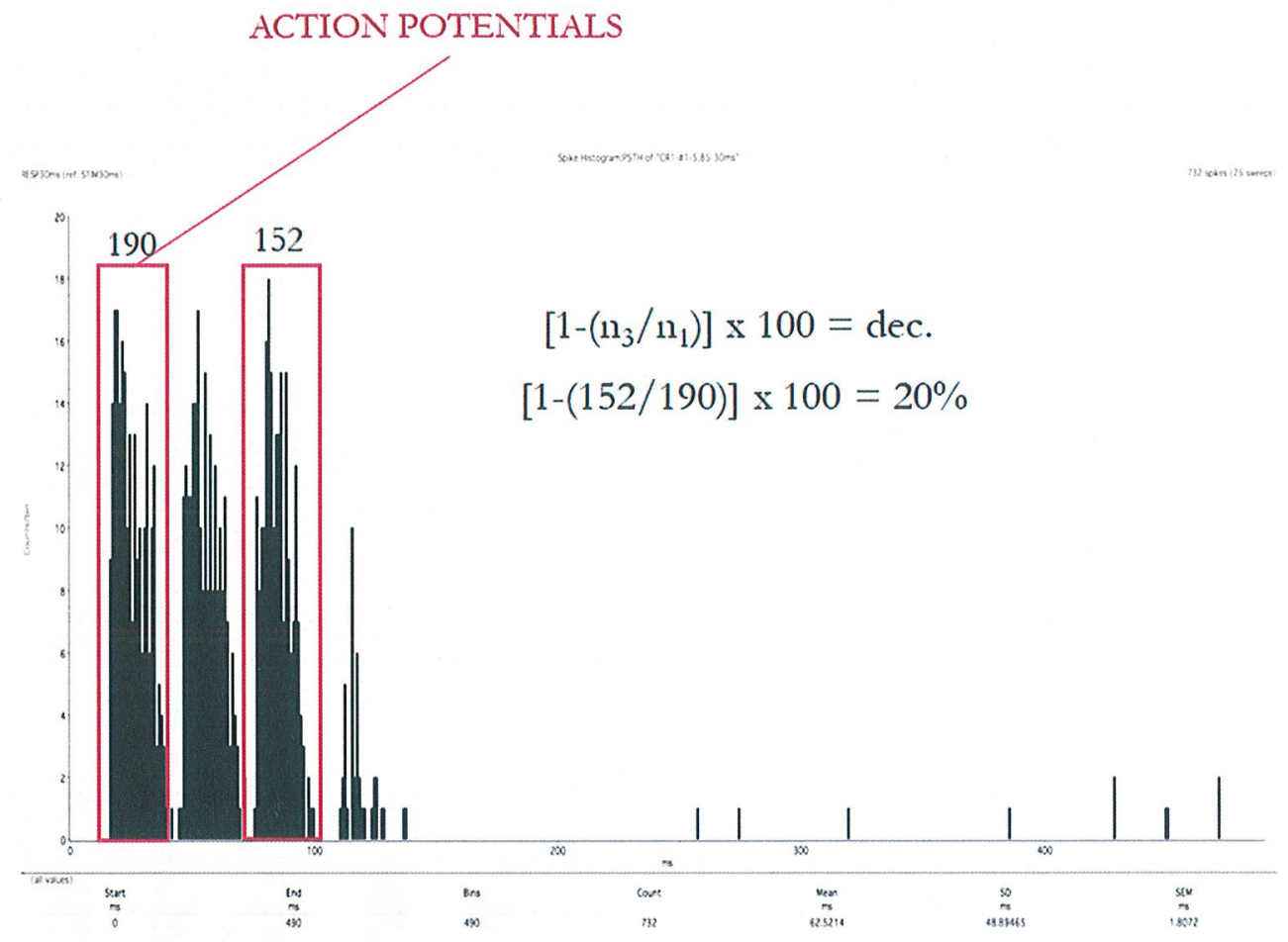

Figure 5. Histogram representing the number of action potentials $/ \mathrm{ms}$. Decrement is calculated by using the formula $\left[1-\left(n_{3} / n_{1}\right)\right] \times 100$, where $n_{1}$ and $n_{3}$ equal the number of action potentials in response to the first and last chirp, respectively. Thus, we can compare these decrement values to the behavioral results in order to establish a relationship between the two.
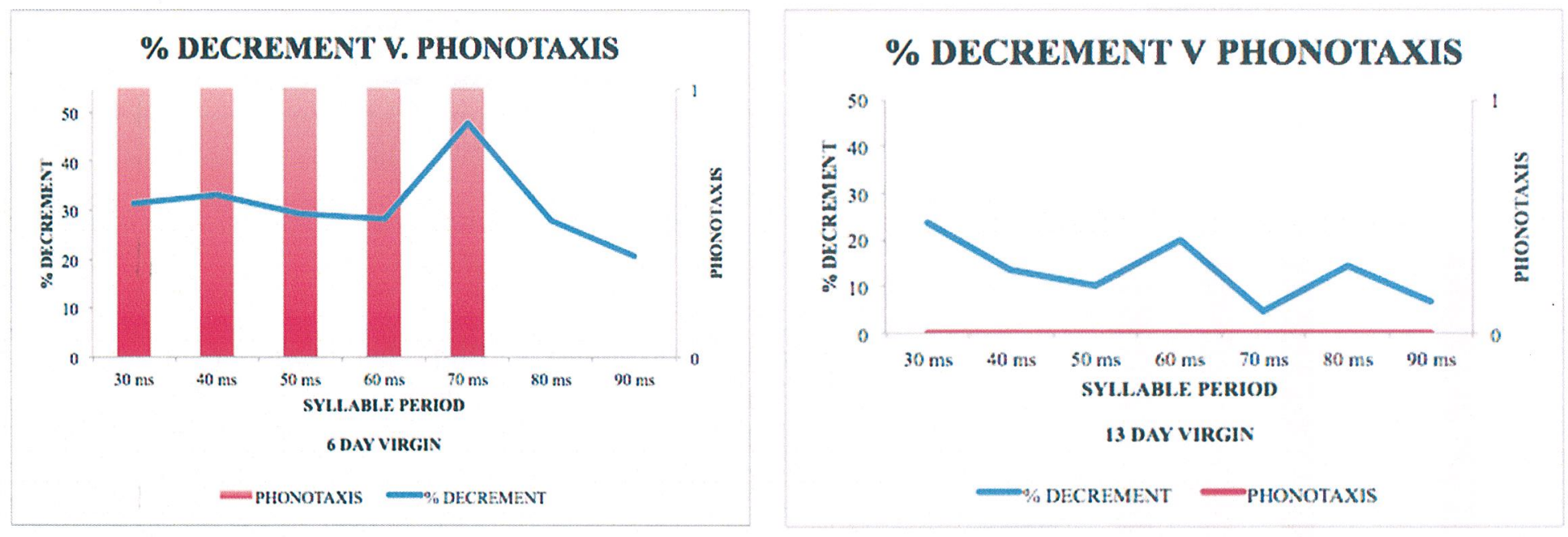

Figure 6. Graphs of the relationship between phonotaxis and \% decrement in a sample of individual crickets. Phonotaxis is plotted in red (right y-axis) as either a 1 - which corresponds to positive phonotaxis, or a 0 - which corresponds to negative phonotaxis. The decrement is plotted in blue (left y-axis) and illustrates the percentage of decrement the cricket expresses that corresponds to each syllable period. A. Six-day virgin female B. Thirteen-day virgin female 

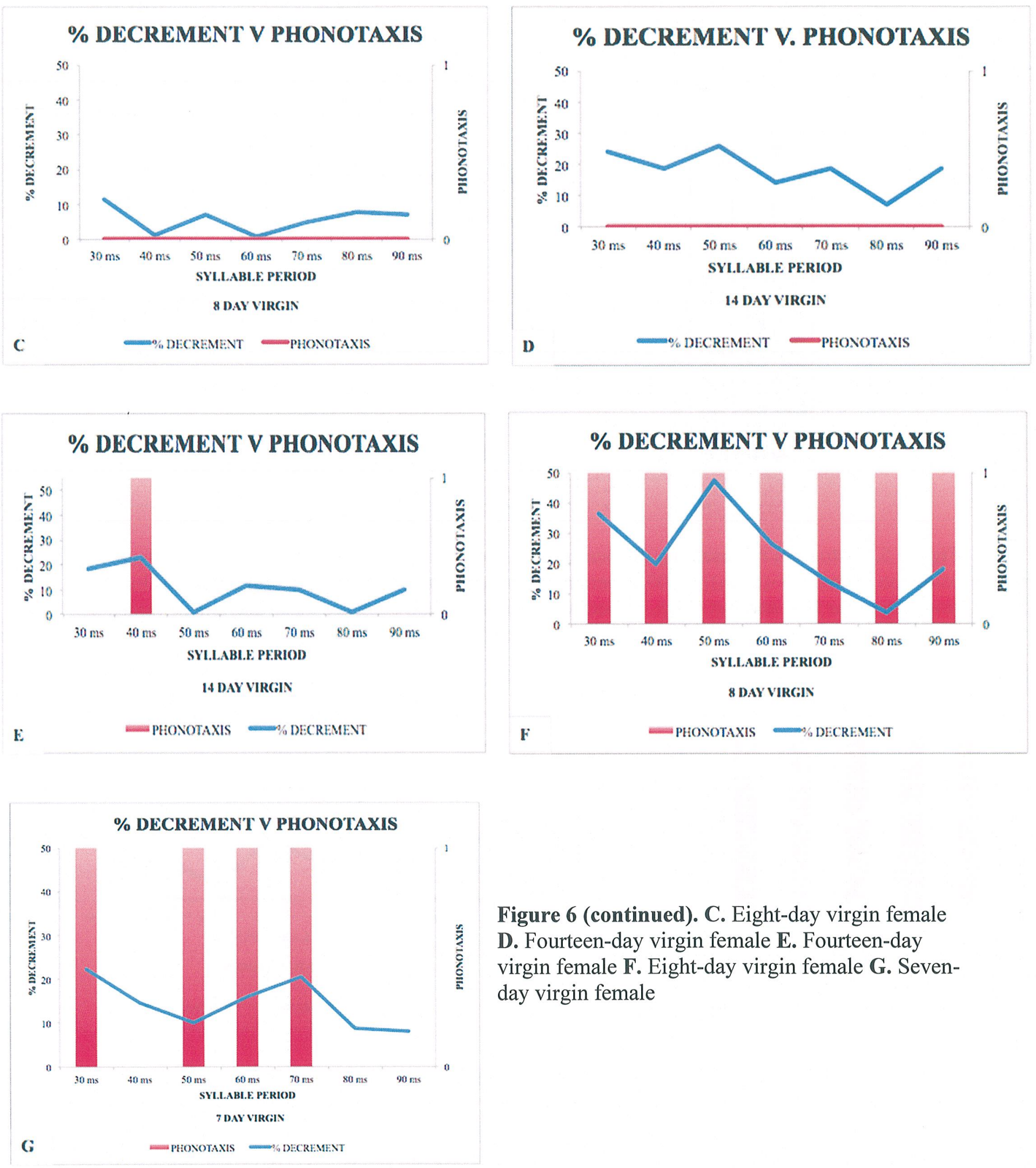

Figure 6 (continued). C. Eight-day virgin female D. Fourteen-day virgin female $\mathbf{E}$. Fourteen-day virgin female $\mathbf{F}$. Eight-day virgin female G. Sevenday virgin female 


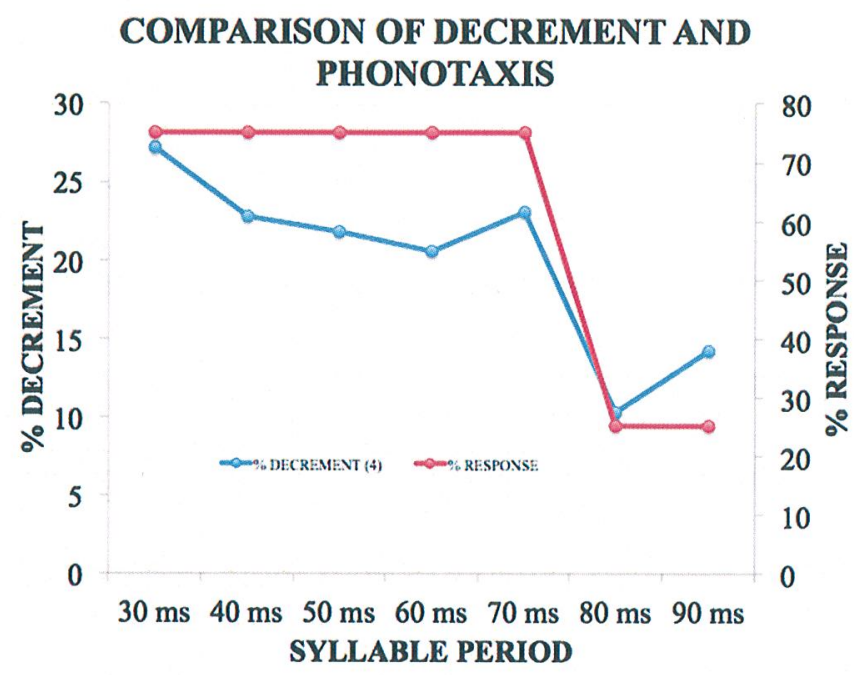

Figure 7. Relationship between average decrement levels (blue) and percentage of crickets (red) which responded to each syllable period over the responsive sample of crickets. This data illustrates higher levels of both positive phonotaxis and decrement between 30-70 ms SPs, while syllable periods of 80-90 ms exhibit lower levels of decrement and responsiveness. Higher levels of decrement correspond to the syllable periods to which crickets respond positively, indicating a possible relationship between decrement and phonotaxis.

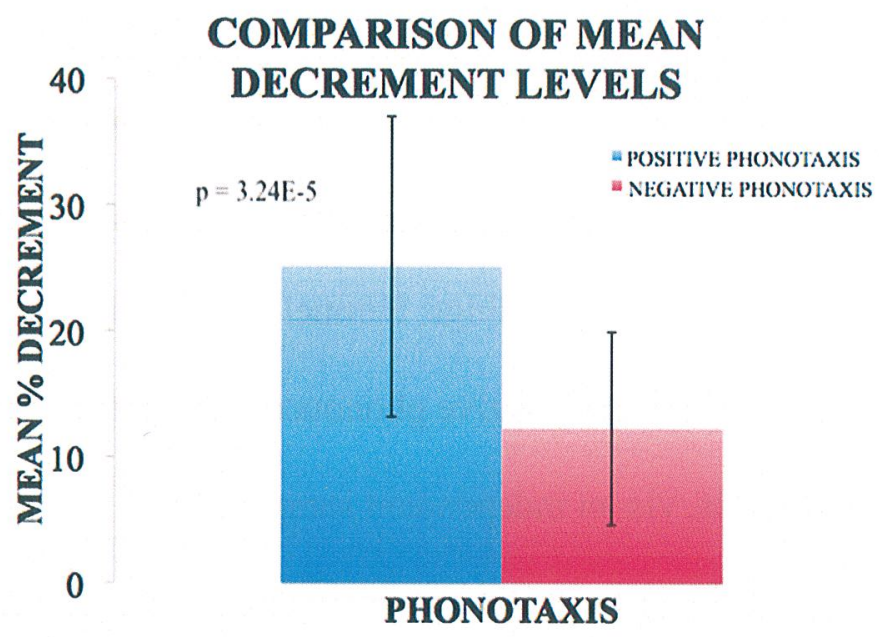

Figure 8. Relationship between the average levels of decrement corresponding to positive phonotaxis and the average levels of decrement corresponding to negative phonotaxis. The blue column represents positive phonotaxis, while the red column represents negative. A total of seven females were tested, thus, an independent sample t-test reveals significant differences between the average levels of decrement of the two groups $\left(t(47)=-4.3856, p=3.24 \times 10^{-5}\right)$. 


\section{Discussion}

These results clearly indicate a relationship between phonotactic behavior and the levels of decrement in the L3 neuron in individual crickets of Acheta domesticus; specifically, each cricket responds with higher levels of decrement to the syllable periods to which it exhibits positive phonotactic behavior, and lower levels of decrement to the SPs to which it exhibits negative phonotactic behavior. For instance, crickets appear to exhibit positive phonotaxis and higher levels of decrement to $30-70 \mathrm{~ms}$ syllable periods more readily than to 80 and $90 \mathrm{~ms}$ (Figure 7). Although throughout the course of the experiment, there were differences in the individual responsiveness of the crickets, (e.g., some crickets responded to all the syllable periods, while others responded to none) when the sample is examined as a whole, the differences between the levels of decrement for positive and negative phonotaxis become apparent.

These conclusions make this study unique in its scope. Past studies have demonstrated a relationship between decrement and phonotaxis in individual crickets in another species of cricket, Gryllus bimaculatus (Samuel et al., 2013), but no such relationship has been shown in Acheta domesticus until now. When the results of the current study are compared to those of Samuel et al. (2013), they exhibit similar findings: Samuel et al. (2013) also found statistically significant greater levels of decrement in positive phonotaxis compared to the levels of decrement in negative phonotaxis, via an independent sample t-test.

However, evaluation of the phonotactic responsiveness of individual crickets in response to particular SPs and their corresponding decrement levels indicates that an individual animal's highest levels of decrement do not always correspond to the syllable periods to which it responds positively. These discrepancies between behavioral and neuronal responses are labelled as 
positively. These discrepancies between behavioral and neuronal responses are labelled as "mismatches," which are defined as when the female either exhibited positive phonotaxis and a low L3 decrement level, or negative phonotaxis and a high L3 decrement level, in response to a particular SP.

These individual mismatches were also observed in the Samuel et al. (2013) study, where 22 mismatches (out of 208 comparisons) indicated that females had exhibited high decrement from its AN2 neuron and negative phonotaxis, or low decrement from its AN2 neuron and positive phonotaxis, in response to a particular SP. These fluctuations in responsiveness both between and within the crickets' responses can be explained by the plasticity in phonotactic choices made by individual females (Stout et al., 2010), as well as the environmental temperature (Navia et al., 2015), hormone levels of the female (Choi et al., 2012), and age of the female (Stout et al., 2010). However, due to the small sample size tested in this study, conclusive statements regarding mismatches cannot be made. Future studies may benefit from utilizing an increased sample size to determine the rate of mismatches between decrement and phonotaxis in A. domesticus. Furthermore, in this study, environmental temperature was maintained at a consistent level between all crickets tested, as well as between the behavioral and neuronal testing procedures, therefore, this factor most likely did not affect the variability in responsiveness.

In addition, Samuel et al. (2013) split the responding animals into two categories: selective for SPs or unselective, in order to further examine whether or not this pattern persisted amongst specific groups of crickets; however, they tested sixty-eight total animals, whereas this 
study tested seven. Thus, due to the smaller sample size, statistical testing between selective and unselective groups in this experiment did not provide conclusive results in our experimentation.

The presence of this relationship between decrement and phonotactic behavior in Acheta domesticus implies the possibility that other features of cricket behavior which have only been investigated in other species, such as G. bimaculatus, can be generalized and applied. In addition, by indicating that crickets responding with positive phonotaxis show statistically significant greater decrement levels than those responding with negative phonotaxis, the results of this study provide a possible basis for the use of decrement percentages to predict the behavior of a cricket in response to a particular SP; if the decrement percentages are known, then the phonotactic behavior of the cricket (either positive or negative) in response to that same syllable period can be inferred with reasonable assurance.

Future investigations should apply this same methodology to older crickets as well as male-exposed female crickets of $A$. domesticus. Both of these groups have demonstrated differences in their selectivity to the computer-generated model calls; thus, by examining the relationship between phonotaxis and decrement in different cricket populations, the pattern exhibited in virgin females of $A$. domesticus can be generalized in its application to other cricket groups and species, such as Gryllus pennsylvanicus or Gryllus veletis ${ }^{1}$. In addition, further testing of virgin females of this species may reveal further findings regarding age-dependent selectivity, as well the syllable periods which appear to be most attractive, both neuronally and behaviorally.

\footnotetext{
${ }^{1}$ The phonotactic selectivity of both these species was tested by Stout et al. (2010); however, their neuronal responsiveness has not yet been investigated.
} 


\section{Literature Cited}

Atkins et al. (1989). Influence of syllable period on song encoding properties of an ascending auditory interneuron in the cricket Acheta domesticus. Journal of Comparative Physiology A: Neuroethology, Sensory, Neural, and Behavioral Physiology, 165, 827 836.

Choi, R., Atkins, G., Stout, J. (2012) The effects of injecting Juvenile Hormone III into the prothoracic ganglion on phonotaxis by female crickets Gryllus bimaculatus. Physiol. Entomol. 37:201-2015.

Huber, F., Thorson, J. (1985) Cricket auditory communication. Scientific American, 253(6): 60-68.

Jeffery, J., Navia, B., Atkins, G., Stout J. (2005). Selective processing of calling songs by auditory interneurons in the female cricket, Gryllus pennsylvanicus: Possible roles in behavior. J. Exp. Zoology. 303(5):377-92.

Navia, B., Stout, J., Atkins G. (2003). Prolonged response to calling songs by the L3 auditory interneuron in female crickets (Acheta domesticus): Intracellular evaluation. J. Exp. Zool. 269A: 63-71.

Navia, B., Burden, C., Steely, T., Hasegawa, H., Cha, E., Henson, S.M., Stout, J., Atkins, G. (2015) Parallel effects of temperature on the male cricket calling song, phonotaxis of the female and the auditory responses of the L3 neurone. Physiol. Entomol. 40:113-122.

Samuel, L., Stumpner, A., Atkins, G. and Stout, J. (2013) Processing of model calling songs by the prothoracic AN2 neurone and phonotaxis are significantly correlated in individual female Gryllus bimaculatus. Physiol. Entomol. 38:344-354.

Stout, J., Navia, B., Jeffery, J., Samuel, L., Hartwig, L., Butlin, A., Chung, M., Wilson, J., Dashner, E., Atkins, G. (2010) Plasticity of the phonotactic selectiveness of four species of chirping crickets (Gryllidae): Implications for call recognition. Physiol. Entomol. 35:99-116.

Stout, J., Stumpner, A., Jeffery, J., Samuel, L., Atkins, G. (2011) Response properties of the prothoracic AN2 auditory interneurone to model calling songs in the cricket Gryllus bimaculatus. Physiol. Entomol. 36:343-359. 